\title{
Random k-labelsets method for human activity recognition with multi-sensor data in smart home
}

\begin{abstract}
In today's world, we are surrounded by ambient sensors everywhere that record our data of activities of daily living. Moreover, the solutions to various applications such as health care, surveillance, home monitoring, and so on are possible by inferring this data. Thus, human activity recognition, especially in the smart home environment, has been a very actively researched problem. Multiple residents in a single home environment pose several challenges making multi-resident activity recognition a daunting task. Therefore, in this paper, we model the Random k-Labelsets method of the Multi-Label Classification to tackle this activity recognition problem. The proposed method not only takes label dependencies into account which is essential for multi-resident activity recognition but also overcomes the drawbacks of other problem transformation methods. Experiments are carried on a real smart home dataset and accuracy, precision and hamming loss are selected as metrics for evaluating the results of the proposed method.
\end{abstract}

Keyword: Multi-label classification; Human activity recognition; Random k-labelsets; Smart home environment 